\title{
Solutions to the cosmological constant problems
}

\author{
J. Garriga \\ Departament de Física, IFAE, Universitat Autonoma de Barcelona, 08193 Bellaterra (Barcelona), Spain \\ and Department of Physics and Astronomy, Institute of Cosmology, Tufts University, Medford, Massachusetts 02155
}

A. Vilenkin

Department of Physics and Astronomy, Institute of Cosmology, Tufts University, Medford, Massachusetts 02155

(Received 23 December 2000; published 22 June 2001)

\begin{abstract}
We critically review several recent approaches to solving the two cosmological constant problems. The "old" problem is the discrepancy between the observed value of $\rho_{\Lambda}$ and the large values suggested by particle physics models. The second problem is the "time coincidence" between the epoch of galaxy formation $t_{G}$ and the epoch of $\Lambda$ domination $t_{\Lambda}$. It is conceivable that the "old" problem can be resolved by fundamental physics alone, but we argue that in order to explain the "time coincidence" we must account for anthropic selection effects. Our main focus here is on the discrete- $\Lambda$ models in which $\Lambda$ can change through nucleation of branes. We consider the cosmology of this type of model in the context of inflation and discuss the observational constraints on the model parameters. The issue of multiple brane nucleation raised by Feng et al. is discussed in some detail. We also review continuous- $\Lambda$ models in which the role of the cosmological constant is played by a slowly varying potential of a scalar field. We find that both continuous and discrete models can in principle solve both cosmological constant problems, although the required values of the parameters do not appear very natural. M-theory-motivated brane models, in which the brane tension is determined by the brane coupling to the four-form field, do not seem to be viable, except perhaps in a very tight corner of the parameter space. Finally, we point out that the time coincidence can also be explained in models where $\Lambda$ is fixed, but the primordial density contrast $Q=\delta \rho / \rho$ is treated as a random variable.
\end{abstract}

DOI: 10.1103/PhysRevD.64.023517

PACS number(s): 98.80.Cq, 11.27.+d

\section{INTRODUCTION}

The cosmological constant $\Lambda$ presents us with at least two intriguing problems. Particle physics models suggest that the natural value for this constant is set by the Planck scale, $M_{P} \sim 10^{18} \mathrm{GeV}$ [we use the reduced Planck mass $M_{P}$ $\left.=(8 \pi G)^{-1 / 2}\right]$. The corresponding vacuum energy density is $\rho_{\Lambda} \sim M_{P}^{4}$, which is some 120 orders of magnitude greater than the observational bounds. In supersymmetric theories, one can expect a lower value,

$$
\rho_{\Lambda} \sim \eta_{S U S Y}^{4}
$$

where $\eta_{S U S Y}$ is the supersymmetry breaking scale. However, with $\eta_{S U S Y} \gtrsim 1 \mathrm{TeV}$, this is still 60 orders of magnitude too high. Until recently, this discrepancy between the expected and observed values was the only cosmological constant problem. Its solution, many believed, was that something so small could only be zero, due to some unknown symmetry or dynamical cancellation.

Thus, it came as a surprise when recent observations [1] provided evidence that the universe is accelerating, rather than decelerating, suggesting a non-zero cosmological constant. ${ }^{1}$ The observationally suggested values of $\Lambda$ correspond to $\rho_{\Lambda} \sim \rho_{M 0}$, where $\rho_{M 0}$ is the present density of matter. This brings yet another puzzle. It is difficult to understand why we happen to live at the epoch when $\rho_{M} \sim \rho_{\Lambda}$. Another statement of the problem is why the time when $\Lambda$ starts dominating the universe nearly coincides with the epoch of galaxy formation,

$$
t_{\Lambda} \sim t_{G}
$$

This is the so-called cosmic coincidence problem.

A number of proposed solutions to these problems have recently appeared in the literature [4-9]. Some of them rely on some form of the anthropic principle, while others do not. To our knowledge, the only approach that can explain both puzzles is the one that attributes them to anthropic selection

\footnotetext{
${ }^{1}$ The surprise, however, was not total. In Ref. [2] (well before the supernova data [1] would give the first observational evidence in this direction) it was noted that anthropic selection effects would place the cosmological constant in the range $\rho_{\Lambda} / \rho_{M 0} \lesssim 10$, and that "the actual value is likely to be comparable to this upper bound." For a flat universe this implies $\Omega_{\Lambda} \sim 0.9$, not far from the observed value and certainly compatible with it, within the accuracy of the prediction. Similar predictions where made in [3] at about the same time.
} 
effects. In this approach, the cosmological constant is assumed to be a random variable that can take different values in different parts of the universe.

The purpose of this paper is to give a critical analysis of the proposed approaches, both anthropic and otherwise. Our main focus will be on the models with a discrete spectrum of $\Lambda$ which have recently attracted much attention. We shall consider these models in the framework of inflationary cosmology and discuss the calculation of the probability distribution for $\rho_{\Lambda}$, as well as the observational constraints on the model parameters.

The paper is organized as follows. In Sec. II we review the motivation for considering $\Lambda$ as a random variable. In Sec. III we discuss models where $\Lambda$ is a discrete variable, in particular the models where there is a four-form contribution to the cosmological constant, which may relax to a small value through nucleation of branes. In Sec. IV we analyze the cosmology of such models. In Sec. V we consider the possibility of coincident brane nucleation. In Sec. VI we discuss models where the cosmological constant is a continuous variable. In Sec. VII we consider the possibility of a slowly varying four-form field in theories with extra dimensions. In Sec. VIII we review some non-anthropic approaches to the problem. In Sec. IX we consider models where the time coincidence is explained by assuming that the primordial density contrast $Q=\delta \rho / \rho$ (and not necessarily $\Lambda$ ) is a random variable. Our conclusions are summarized in Sec. X.

\section{II. $\Lambda$ AS A RANDOM VARIABLE}

Not all values of $\Lambda$ are consistent with the existence of conscious observers. This observation was made by Barrow and Tipler [10] (see also [11]), but the first quantitative analysis is due to Weinberg [12]. In a spatially flat universe with a cosmological constant, gravitational clustering effectively stops at $t \sim t_{\Lambda}$. At later times, the vacuum energy dominates and the universe enters a de Sitter stage of exponential expansion. An anthropic bound on $\rho_{\Lambda}$ can be obtained by requiring that it does not dominate before the redshift $z_{\max }$ when the earliest galaxies are formed. Weinberg took $z_{\max } \sim 4$ and obtained

$$
\rho_{\Lambda} \lesssim 100 \rho_{M 0} .
$$

This is a dramatic improvement over Eq. (1), but it still falls short of the observational bound by a factor of about 30 .

The anthropic bound (3) specifies the value of $\rho_{\Lambda}$ which makes galaxy formation barely possible. However, as it was pointed out in $[2,3]$, the observers are where the galaxies are, and thus most of the observers will detect not these marginal values, but rather the values that maximize the number of galaxies. More precisely, the probability distribution for $\rho_{\Lambda}$ can be written as

$$
d \mathcal{P}\left(\rho_{\Lambda}\right)=\mathcal{P}_{*}\left(\rho_{\Lambda}\right) \nu\left(\rho_{\Lambda}\right) d \rho_{\Lambda} .
$$

Here, $\mathcal{P}_{*}\left(\rho_{\Lambda}\right) d \rho_{\Lambda}$ is the a priori distribution, which is proportional to the volume of those parts of the universe where $\rho_{\Lambda}$ takes values in the interval $d \rho_{\Lambda}$, and $\nu\left(\rho_{\Lambda}\right)$ is the average number of galaxies that form per unit volume with a given value of $\rho_{\Lambda}$. According to the "principle of mediocrity," which assumes that we are typical observers, Eq. (4) gives the probability distribution for us to observe a given value of $\rho_{\Lambda}$. The calculation of $\nu\left(\rho_{\Lambda}\right)$ is a standard astrophysical problem; it can be done, for example, using the Press-Schechter formalism [13]. The a priori distribution $\mathcal{P}_{*}\left(\rho_{\Lambda}\right)$ should be determined from the theory of initial conditions, e.g., from an inflationary model.

Martel, Shapiro and Weinberg [14] (see also [15]) presented a detailed calculation of $d \mathcal{P}\left(\rho_{\Lambda}\right)$ assuming a flat $a$ priori distribution,

$$
\mathcal{P}_{*}\left(\rho_{\Lambda}\right)=\text { const }
$$

in the range of interest (3). They found that the peak of the resulting probability distribution is close to the observationally suggested values of $\rho_{\Lambda}$. The cosmic time coincidence is easy to understand in this approach $[16,17]$ if one notes that regions of the universe where $t_{\Lambda} \ll t_{G}$ do not form any galaxies at all, whereas regions where $t_{\Lambda} \gg t_{G}$ are suppressed by "phase space," since they correspond to a very tiny range of $\Lambda$. It was shown in Ref. [16] that the probability distribution for $t_{G} / t_{\Lambda}$ is peaked at $t_{G} / t_{\Lambda} \approx 1.5$, and thus most observers will find themselves in galaxies formed at $t_{G} \sim t_{\Lambda}$.

This anthropic solution to the cosmological constant problems is incomplete without a particle physics model that would allow $\Lambda$ to take different values and without a theory of initial conditions, such as an inflationary cosmological model, that would allow one to calculate the a priori distribution $\mathcal{P}_{*}\left(\rho_{\Lambda}\right)$.

One possibility is to consider models in which the role of the vacuum energy is played by a slowly varying potential $V(\phi)$ of some scalar field $\phi$, which is very weakly coupled to ordinary matter. The values of $\phi$ are randomized by quantum fluctuations during inflation, and analysis shows that the resulting a priori distribution is indeed flat for a wide class of potentials $[4,18]$. The main challenge one has to face in this approach is to justify the exceedingly flat potential $V(\phi)$ required by the model. We shall comment on this issue in Sec. VI. Before that, we shall consider an alternative possibility which has recently attracted much attention. This is provided by models with a discrete spectrum of $\rho_{\Lambda}$.

\section{MODELS WITH A DISCRETE SPECTRUM OF $\Lambda$}

The first model of this type was suggested in an early paper by Abbott [19] as an attempt to solve the old cosmological constant problem. He considered a self-interacting scalar field $\phi$ with a "washboard" potential $V(\phi)$ of the form illustrated in Fig. 1. The potential has local minima at $\phi_{n}=n \eta$ with $n=0, \pm 1, \pm 2, \ldots$, separated from one another by barriers. The vacuum at $\phi=\phi_{n}$ has energy density

$$
\rho_{\Lambda n}=n \epsilon+\text { const }
$$

and can decay through bubble nucleation to the vacuum at $\phi_{n-1}$.

The nucleation rate $\Gamma_{n \downarrow}$ per unit spacetime volume is given by 


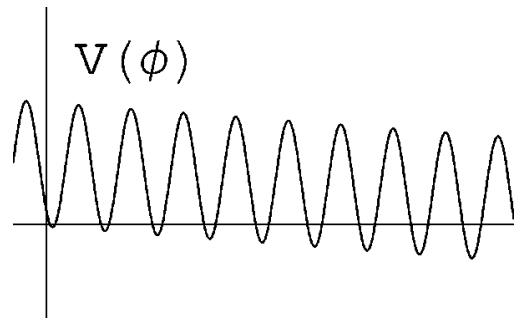

FIG. 1. The washboard potential.

$$
\Gamma_{n \downarrow}=A_{n} e^{-B_{n}},
$$

where $B_{n}$ is the action of the Coleman-de Luccia instanton [20] and the meaning of the subscript $\downarrow$ will become clear shortly. The bubble radius at nucleation $R_{n}$ is bounded by $0<R_{n}<H_{n}^{-1}$, where

$$
H_{n}^{2}=\frac{\rho_{n}}{3 M_{P}^{2}}
$$

is the square of the expansion rate of de Sitter space filled with the vacuum $\phi_{n}$. The horizon radius and the curvature radius of that space are both equal to $H_{n}^{-1}$.

An analytic expression for $B_{n}$ can be given in the thin wall approximation, when $\delta \ll R_{n}$ [20]. The general expression is somewhat cumbersome and we shall only consider the limiting cases of interest. For $R_{n} \ll H_{n}^{-1}, B_{n}$ is given by the flat space expression [21]

$$
B_{n}^{(\text {flat })} \approx \frac{27 \pi^{2}}{2} \frac{\sigma^{4}}{\epsilon^{3}}
$$

approximately independent of $n$. In this regime $R_{n} \approx 3 \sigma / \epsilon$, so we should have $\sigma H_{n} / \epsilon \ll 1$. In the opposite limit, $\sigma H_{n} / \epsilon \gg 1$, we have $\left(H_{n}^{-1}-R_{n}\right) \ll H_{n}^{-1}$ and

$$
B_{n}^{(w a l l)} \approx 2 \pi^{2} \sigma H_{n}^{-3} .
$$

The vacuum energy difference $\epsilon$ is unimportant in this case, and the action coincides with that for domain wall nucleation [22]. The prefactor in Eq. (7) can be estimated as (see e.g. [23])

$$
A_{n} \sim \sigma^{2} R_{n}^{2}
$$

Equations (9)-(11) apply under the condition that the gravitational effect of the wall is negligible,

$$
\sigma \ll M_{P}^{2} H_{n}
$$

Upward quantum jumps from $\phi_{n-1}$ to $\phi_{n}$ are also possible [24]. The corresponding nucleation rate is

$$
\Gamma_{(n-1) \uparrow}=\exp \left[24 \pi^{2} M_{P}^{4}\left(\frac{1}{\rho_{n}}-\frac{1}{\rho_{n-1}}\right)\right] \Gamma_{n \downarrow} .
$$

For $\epsilon \ll \rho_{n}$ this can be approximated as

$$
\Gamma_{(n-1) \uparrow}=\exp \left(-\frac{8 \pi^{2}}{3} \frac{\epsilon}{H_{n}^{4}}\right) \Gamma_{n \downarrow},
$$

where we have used Eq. (8) for $H_{n}$.

In order for the anthropic explanation to work, one needs

$$
\epsilon \lesssim \rho_{M 0} \sim\left(10^{-3} \mathrm{eV}\right)^{4},
$$

and in order to have successful baryogenesis, the energy density during inflation should exceed $(1 \mathrm{TeV})^{4}$, which corresponds to

$$
H \gtrsim 10^{-3} \mathrm{eV} \text {. }
$$

Combining this with Eq. (14), we see that the probabilities of upward and downward jumps in $\rho_{\Lambda}$ during inflation are nearly equal, except perhaps in the borderline case when

$$
H \sim \epsilon^{1 / 4} \sim 10^{-3} \mathrm{eV}
$$

An alternative discrete model, first discussed by Brown and Teitelboim [25], assumes that the cosmological constant is due to a four-form field,

$$
F^{\alpha \beta \gamma \delta}=\frac{F}{\sqrt{-g}} \epsilon^{\alpha \beta \gamma \delta},
$$

which can change its value through the nucleation of branes. The total vacuum energy density is given by

$$
\rho_{\Lambda}=\rho_{\text {bare }}+F^{2} / 2 \text {, }
$$

where $\rho_{\text {bare }}<0$ is the "bare" cosmological constant at $F$ $=0$. The change of the field strength across the brane is

$$
\Delta F= \pm q,
$$

where $q=$ const is fixed by the model. The four-form model has recently attracted much attention because four-form fields with appropriate couplings to branes naturally arise in the context of $\mathrm{M}$ theory. In this case the brane tension is $[5,6]$

$$
\sigma=q M_{P} / \sqrt{2}
$$

and the effective thickness of the branes is $\delta \sim M_{P}^{-1}$, so that the thin wall approximation is justified.

At present we should have $|F| \approx\left(-2 \rho_{\text {bare }}\right)^{1 / 2}$, so that the bare cosmological constant is almost neutralized. Then, in the range of interest, the spectrum of $\rho_{\Lambda}$ is nearly equidistant, with a separation

$$
\Delta \rho_{\Lambda} \equiv \epsilon \approx\left(-2 \rho_{\text {bare }}\right)^{1 / 2} q,
$$

and the model is very similar to the Abbott's "washboard", model. We expect

$$
\left|\rho_{\text {bare }}\right| \gtrsim(1 \mathrm{TeV})^{4} \text {, }
$$

and it follows from Eq. (15) that $q \lesssim 10^{-90} M_{P}^{2}$ and

$$
\sigma \lesssim\left(10^{-3} \mathrm{eV}\right)^{3},
$$


where we have used the relation (21) between $\sigma$ and $q$. Such small values of $q$ and $\sigma$ may appear problematic, but in a recent paper [6] Feng, March-Russell, Sethi and Wilczek (FMSW) have argued that they can naturally arise due to non-perturbative effects in M theory. With $\sigma$ and $H$ satisfying the bounds (24) and (16), the condition of negligible brane gravity (12) is also satisfied, and thus Eqs. (9),(10) can be used.

With the aid of Eqs. (21)-(24) it can be easily verified that the flat space bounce action (9) is bounded by [6]

$$
B^{(f l a t)} \lesssim 10^{2}
$$

This inequality is saturated for $\rho_{\text {bare }} \sim(1 \mathrm{TeV})^{4}$ and

$$
\epsilon^{1 / 4} \sim \sigma^{1 / 3} \sim 10^{-3} \mathrm{eV}
$$

If $\sigma$ and $\epsilon$ significantly differ from these borderline values, then $B \leqq 1$ and brane nucleation is unsuppressed. A similar bound is obtained for the wall nucleation action (10) using Eqs. (24) and (16):

$$
B^{(\text {wall })} \lessgtr 20 .
$$

Here, the inequality is saturated for

$$
H \sim \sigma^{1 / 3} \sim 10^{-3} \mathrm{eV} .
$$

We note that Eqs. (21),(22) apply only to models based on M theory, and therefore the constraints (24), (25), and (27) are also limited to this class of models.

A different version of the four-form model has been developed by Bousso and Polchinski (BP) [5]. They assume that several four-forms $F_{i}$ are present, so that Eq. (19) is replaced by

$$
\rho_{\Lambda}=\rho_{\text {bare }}+\frac{1}{2} \sum_{i} F_{i}^{2} .
$$

The corresponding "charges" $q_{i}$ are not assumed to be very small, but BP have shown that with multiple four-forms the spectrum of the allowed values of $\rho_{\Lambda}$ can be sufficiently dense to satisfy the condition (15) in the range of interest. However, the situation here is quite different from that in the FMSW model. As pointed out by the authors themselves, and further emphasized by Banks, Dine and Motl [26], the vacua with nearby values of $\rho_{\Lambda}$ have very different values of $F_{i}$ and are expected to have very different physical properties. There is no reason to expect the a priori probabilities for these vacua to be similar. Moreover, the low energy physics in different vacua is likely to be different, so the process of galaxy formation and the types of life that can evolve will also differ. It appears therefore that the anthropic approach to solving the cosmological constant problems cannot be applied to this case [26].

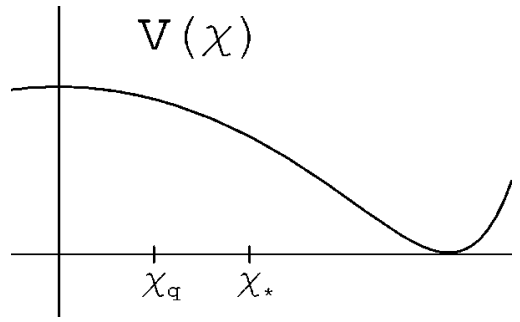

FIG. 2. Inflaton potential.

\section{COSMOLOGY OF THE FOUR-FORM MODELS}

\section{A. A priori distribution}

We shall now discuss the four-form models with brane nucleation in the context of inflationary cosmology. The energy density of the universe during inflation can be expressed as

$$
\rho_{n}(\chi)=U(\chi)+\rho_{\Lambda n},
$$

where $U(\chi)$ is the potential of the inflaton field $\chi, \rho_{\Lambda n}$ is the cosmological constant contribution (19), and index $n$ labels the vacuum energies corresponding to different values of the four-form field $F$. [The inflaton potential is generally $F$-dependent and has different forms $U_{n}(\chi)$ in different vacua $[5,26]$. Here we shall disregard this difference, assuming that the variation of $U(\chi)$ is negligible in the narrow anthropic range of $\rho_{\Lambda}$ that will be of interest to us.] The minimum of $U(\chi)$ is assumed to be at $U_{\min }=0$. The spacetime during inflation is locally approximately de Sitter,

$$
d s^{2}=d t^{2}-e^{2 H_{n} t} d \mathbf{x}^{2},
$$

with $H_{n}(\chi)$ given by Eq. (8).

A remarkable feature of inflation, which will play an important role in our discussion here, is that generically inflation never ends completely in the entire universe. The evolution of the inflaton field $\chi$ is influenced by quantum fluctuations, and as a result thermalization does not occur simultaneously in different parts of the universe. In most of the models, one finds that at any time there are parts of the universe that are still inflating and that the total volume of inflating regions is growing with time $[27,28]$. This picture is often referred to as stochastic, or eternal, inflation.

The full range of the field $\chi$ can be divided into the "diffusion," slow-roll, and thermalization parts, as illustrated in Fig. 2. In the diffusion range, $\chi \lesssim \chi_{q}$, the inflaton dynamics is dominated by quantum fluctuations. It is this regime that is responsible for the eternal nature of inflation. In the slow-roll regime, $\chi_{q} \lesssim \chi \lesssim \chi_{*}$, the inflaton rolls down its potential. As it reaches the thermalization point $\chi_{*}$, it starts oscillating about the minimum of the potential, and its energy gets thermalized. The hypersurfaces $\chi=\chi_{*}$ are therefore the boundaries between inflating and thermalized regions of spacetime. These surfaces play the role of the big bang for the corresponding thermalized regions. There is typically an infinite number of such surfaces, each of them having an infinite volume. (For a discussion of the spacetime structure of inflationary universe see, e.g., [29].) 
As the inflaton $\chi$ fluctuates, rolls down its potential, and eventually thermalizes its energy, spherical branes nucleate at the rates (7),(13) changing the local values of the fourform field $F$. All possible values of $\rho_{\Lambda n}$ will be taken on each infinite thermalization surface $\Sigma_{*}$, and the a priori probability $\mathcal{P}_{* n}$ can be defined as the fraction of the volume of $\Sigma_{*}$ occupied by regions with vacuum energy density $\rho_{\Lambda n}$. [The volume fraction on an infinite hypersurface can be defined by calculating this fraction in a sphere of geodesic radius $R$ and taking the limit $R \rightarrow \infty$.]

Brane nucleation can both decrease and increase the value of $\rho_{\Lambda n}$; the corresponding nucleation rates are related by Eq. (13). For $\Lambda$-lowering events, the bubble radius is initially smaller than the horizon $H_{n}^{-1}$ and then grows in the comoving coordinates, while for $\Lambda$-raising events the radius is initially larger than the horizon and then decreases in the comoving coordinates. In both cases, with an appropriate definition of the nucleation time, the radius of the bubble nucleated at $t=0$ asymptotically approaches $H_{n}^{-1} e^{H_{n} t}[30]$. This means that the region affected by each nucleation event is a sphere of initial radius $H_{n}^{-1}$. For a comoving observer in vacuum $n$, the probabilities per unit time to witness a $\Lambda$-raising or lowering event are

$$
\begin{gathered}
\kappa_{n \uparrow}=\Gamma_{n \uparrow} \frac{4 \pi}{3} H_{n}^{-3}, \\
\kappa_{n \downarrow}=\Gamma_{n \downarrow} \frac{4 \pi}{3} H_{n}^{-3} .
\end{gathered}
$$

It follows from Eq. (13) that these probabilities are related by

$$
\kappa_{(n-1) \uparrow}=\kappa_{n \downarrow}\left(f_{n-1} / f_{n}\right),
$$

where

$$
f_{n}=H_{n}^{-3} \exp \left(-\frac{24 \pi^{2} M_{P}^{4}}{\rho_{n}}\right)
$$

Consider an ensemble of comoving observers and let $p_{n}(t)$ be the fraction of observers in the $n$th vacuum, where $t$ is the proper time along the observers' world lines. The time evolution of $p_{n}$ is described by the equations

$$
d p_{n} / d t=-\left(\kappa_{n \uparrow}+\kappa_{n \downarrow}\right) p_{n}+\kappa_{(n-1) \uparrow} p_{n-1}+\kappa_{(n+1) \downarrow} p_{n+1} .
$$

Let us assume for a moment that the inflaton potential remains unchanged,

$$
U(\chi)=\text { const },
$$

so that $\kappa_{n \uparrow}$ and $\kappa_{n \downarrow}$ do not change with time. Then the solutions of Eq. (36) approach the stationary distribution

$$
p_{n} \propto f_{n}^{-1} \propto H_{n}^{3} \exp \left(\frac{24 \pi^{2} M_{P}^{4}}{\rho_{n}}\right) .
$$

We shall be mostly interested in the probability distribution in the anthropic range (3), where $\rho_{\Lambda n}$ can be approximated by Eq. (6) with $\epsilon$ from Eq. (22), and Eq. (38) takes the form

$$
p_{n} \propto \exp \left(-\frac{8 \pi^{2} \epsilon}{3 H^{4}} n\right) \text {. }
$$

If inflation is well above the electroweak scale, $H$ $\gg 10^{-3} \mathrm{eV}$, then the distribution (39) is nearly flat in the anthropic range,

$$
p_{n} \approx \text { const. }
$$

The assumption (37) may or may not be a good approximation, depending on the shape of the potential $U(\chi)$. A simple example of a model where this approximation is adequate is a "new inflation" type model with a very flat potential in the diffusion range near the maximum of $U(\chi)$ and a relatively steep decline to the minimum in the slow roll range. The distribution (38) is established during the very long diffusion period, and then it does not change much during the slow roll period if the duration of the slow roll is shorter than the characteristic bubble nucleation time. Here we shall assume that the approximation (37) is justified.

Can the distribution (38) be identified with the a priori probability distribution $\mathcal{P}_{* n}$ ? The answer is "Yes, but only in a restricted class of models." An ensemble of comoving observers gives a comoving-volume distribution for $\rho_{\Lambda n}$, which does not account for the fact that regions with different values of $\rho_{\Lambda n}$ expand at different rates. The condition for this effect to be negligible is that brane nucleations should reshuffle the values of $\rho_{\Lambda n}$ between different regions on a time scale $\tau_{B}$ which is much shorter than the time $\tau_{H}$ it takes for the differential expansion rate to significantly modify the distribution,

$$
\tau_{B} \ll \tau_{H}
$$

As we noted in Sec. II, the probabilities of upward and downward jumps in $\rho_{\Lambda}$ should be nearly equal, except perhaps in the borderline case (17). This means that the evolution of $\rho_{\Lambda}$ can be pictured as a random walk with steps taken on a timescale $\tau \sim \kappa^{-1} \sim H^{3} \Gamma^{-1}$. The anthropic range (3) comprises $N \sim 10^{2} \rho_{M 0} / \epsilon$ steps, and thus

$$
\tau_{B} \sim N^{2} H^{3} \Gamma^{-1} \sim 10^{4}\left(\frac{\rho_{M 0}}{\epsilon}\right)^{2} \frac{H^{3}}{\sigma^{2} R_{0}^{2}} e^{B},
$$

where we have used Eqs. (7) and (11). [In this discussion we have dropped the subscripts $\uparrow$ and $\downarrow$, since the upward and downward nucleation rates are nearly equal, and the subscript $n$ since $H_{n}$ is nearly constant in the anthropic range.]

The variation of the expansion rate in the anthropic range of $\rho_{\Lambda}$ is

$$
\delta H \sim \frac{N \epsilon}{M_{P}^{2} H},
$$

and the time $\tau_{H}$ can be estimated as 


$$
\tau_{H} \sim 1 / \delta H \sim 10^{-2} M_{P}^{2} H / \rho_{M 0} .
$$

The condition $\tau_{B} \ll \tau_{H}$ can now be expressed as

$$
e^{B} \ll 10^{-6} \frac{\epsilon^{2} \sigma^{2} M_{P}^{2} R_{0}^{2}}{H^{2} \rho_{M 0}^{3}} .
$$

Parameter values satisfying this condition can be readily found.

What happens in the opposite limit, when $\tau_{B} \gg \tau_{H}$ ? In this case the differential expansion is important and the probabilities for faster expanding regions with higher values of $\rho_{\Lambda}$ are strongly enhanced. The predicted values of $\rho_{\Lambda}$ should therefore be significantly higher than those obtained with a flat a priori distribution. Martel et al. [14] have found that in the latter case the probability distribution is peaked at $\rho_{\Lambda}$ $=0$, the width of the peak being somewhat broader than the observationally suggested value. Models with $\tau_{B} \gg \tau_{H}$ will have the peak displaced towards higher values of $\rho_{\Lambda}$ and are therefore unlikely to give a good agreement with observations. A quantitative analysis of probability distributions in such models can be given by a relatively straightforward generalization of the formalism developed in Ref. [30].

We note finally that in models with borderline values of parameters (17) the a priori distribution (39) can significantly deviate from flatness, with smaller values of $\rho_{\Lambda}$ being favored. This would displace the peak of the resulting distribution to negative values of $\rho_{\Lambda}$ and if anything would make the observational situation even worse.

\section{B. Observational constraints}

Models of the type we are discussing here suggest that we live in a bubble surrounded by an expanding brane. The values of $\rho_{\Lambda}$ inside and outside the brane are different. Let us first assume that the visible universe is contained within a single bubble. This means that the brane surrounding our bubble nucleated before the presently observable universe crossed the horizon during inflation. For this situation to be typical, the brane nucleation rates should be rather low, both during inflation and at present. This requires that the corresponding bounce actions should be large, $B \gg 1$. In M-theory motivated models this is possible only for the borderline values of the parameters,

$$
H \sim \epsilon^{1 / 4} \sim \sigma^{1 / 3} \sim 10^{-3} \mathrm{eV} .
$$

However, as we discussed at the end of Sec. IV A, these values seem to be disfavored by observations.

The brane nucleation rate at present is given by Eq. (7) with $B$ and $A$ from Eqs. (9),(11). In order to have no brane nucleations in the observable universe in a Hubble time, we have to require that

$$
\Gamma t_{0}^{4} \lesssim 1
$$

where $t_{0}$ is the present cosmic time. For the parameter values (46), $A \sim\left(10^{-3} \mathrm{eV}\right)^{4}$ and Eq. (47) gives $\exp \left(-B^{(\text {flat })}\right)$ $\lesssim 10^{-116}$, or

$$
B^{(\text {flat })} \gtrsim 270 .
$$

This is only marginally consistent with the bound (25).

The brane nucleation rate during inflation is determined by the smaller of the two bounce actions (9), (10). Equation (46) tells us that in models based on M-theory brane nucleation can be suppressed only if the expansion rate during inflation is $H \sim 10^{-3} \mathrm{eV}$. Let $\mathcal{N} \sim 30$ be the number of e-foldings from the time when the comoving region corresponding to the presently observable universe crossed the horizon to the end of inflation. Then the size of this region at the end of inflation is $H^{-1} e^{\mathcal{N}}$. In order to have no brane nucleations in this region during this whole period, we have to require

$$
\Gamma H^{-4} e^{3 \mathcal{N}} \lesssim 1
$$

For the parameter values (46) this gives $B \gtrsim 90$, again marginally consistent with Eqs. (25),(27).

We thus see that M-theory based four-form models could in principle provide a solution to the cosmological constant problems, but only if inflation is at a TeV scale and $\sigma$ and $\epsilon$ are in the tight corner of the parameter space (46). With such values of the parameters, the condition (45) can be (marginally) satisfied. However, from Eq. (14) we then find a significant bias towards $\Lambda$-lowering nucleation events, which would shift the a priori distribution (39) towards lower $\Lambda$. This would result in a prediction near the lower anthropic bound $\rho_{\Lambda} \sim-\rho_{M 0}$. The bias towards $\Lambda$-lowering events might be compensated to some extent by the differential expansion rate, which adds relative volume to regions with high $\Lambda$. However, both effects are exponential, and unless there is a conspiracy in the parameters of the model, the differential expansion is likely to be either insignificant or dominant. In the latter case, the a priori distribution would be biased towards large $\Lambda$, and it would be likely to predict a cosmological constant much larger than observed. In summary, it seems difficult to obtain a flat a priori distribution even in the range (46). Of course, the possibility cannot be excluded with our order of magnitude estimates, and there may still be a small viable region of parameter space in this borderline range. We note also that for models unrelated to $\mathrm{M}$ theory the allowed parameter space is much larger.

Suppose now that the visible universe contains more than one bubble. This would generally result in microwave background anisotropies of amplitude $\delta T / T \gtrsim \epsilon / \rho_{M 0}$, so to avoid conflict with observations we have to require

$$
\epsilon \lesssim 10^{-5} \rho_{M 0}
$$

This takes us far from the borderline values (46), and thus the multiple bubble scenario cannot be realized in M-theory based models. For non-M-theory models, a suitable set of parameters can be easily found by choosing $\sigma$ and $H$ sufficiently large, while keeping $\epsilon$ under the bound (50).

The multiple bubble scenario is feasible only if branes have negligible interaction with ordinary matter. Otherwise we would see fireworks along the bubble boundaries, where the branes hit the stars and where they hit one another. However, the gravitational impact of the branes cannot be 
avoided. An observer outside an expanding spherical bubble does not experience any gravitational force until he is hit by the brane. While the brane passes through the observer, the part of his body inside the brane will experience an acceleration $a=G M / R^{2}$ relative to the part of the body still outside the brane. Here, $M=(4 \pi / 3) \epsilon R^{3}$ and $R$ is the bubble radius at the moment of impact. With $R \sim t_{0}$ and $\epsilon$ satisfying Eq. (50), we have $a \sim G \epsilon t_{0} \sim\left(\epsilon / \rho_{M 0}\right) t_{0}^{-1} \lesssim 10^{-12} \mathrm{~cm} / \mathrm{s}^{2}$. The relative speed developed during the passage time $\Delta t$ $\sim 10^{-8} \mathrm{~s}$ is $\Delta v \lessgtr 10^{-20} \mathrm{~cm} / \mathrm{s}$, and the corresponding displacement is much smaller than the inter-atomic distance. For a brane passing through a sun-like star, $\Delta t \sim 10 \mathrm{~s}$ and the displacement is still smaller than the atomic scale. Thus, if a brane is to sweep through the solar system, its only effect would be to set up imperceptible vibrations in the objects it leaves behind.

What happens if $B<1$, so that brane nucleation is completely unsuppressed? The main danger here is that the vacuum energy will decay so fast that it will drop significantly in less than a Hubble time. This can be countered by choosing $\epsilon$ so small that the change in $\rho_{\Lambda}$ is negligible even after nucleation of a large number of bubbles. This case, however, is almost indistinguishable from that of a scalar field with a very flat potential, which will be discussed in Sec. VI.

\section{No empty universe problem}

Here we shall comment on the so called empty universe problem which was encountered in all earlier work on discrete $\Lambda$ models $[31,19,25,5,6,9,26]$. The scenario these authors had in mind is that the universe starts with a large cosmological constant and relaxes, within the available cosmic time, to a metastable vacuum with an observationally acceptable value of $\Lambda$. The problem is that, in order to make the present vacuum sufficiently stable, the brane nucleation has to be strongly suppressed. One then finds that the time it takes the universe to evolve to the low-energy vacuum is so large that, by the time when the process is complete, any matter that the universe initially had gets diluted to an extremely low density. So one ends up with an empty universe dominated by the cosmological constant.

A number of solutions to this problem have been proposed. FMSW suggested [6] that the nucleation rate of multiple coincident branes may be enhanced due to the increased density of states. They argued that this would lead to a rapid descent of the vacuum energy towards lower values. To ensure the long lifetime of the present vacuum, they argued that this rate enhancement may not apply to the vacuum with the lowest positive value of $\rho_{\Lambda}$. Bousso and Polchinski [5], who considered brane nucleation with large jumps in $\rho_{\Lambda}$, suggested that the penultimate vacuum could have a high energy density. The inflaton field would then be excited to high values of its potential by quantum fluctuations. When the ultimate brane nucleates, the inflaton rolls down the potential thermalizing its energy and providing a high density of matter. Alternatively, they suggested that the nucleation of the ultimate bubble, which in their model is accompanied by a large change in the four-form field $F$, can be accompanied by a large modification of the inflaton potential. As a result the inflaton will be displaced from the minimum of the potential, even if it was at the minimum prior to the bubble nucleation.

In our view, the empty universe problem is not a real problem, and the attempts to solve it seem therefore unnecessary. The problem disappears when the eternal nature of inflation is taken into account. As the inflaton fluctuates back and forth in the quantum diffusion regime, branes are constantly being nucleated and all possible values of $\rho_{\Lambda}$ are reached. The slow rate of brane nucleation is not a problem, since an unlimited amount of time is available. Thermalization of the inflaton energy occurs at different times in different parts of the universe, and each region inherits the local value of $\rho_{\Lambda}$. Each possible value is represented in the thermalized regions of the universe. We are interested only in those regions where $\rho_{\Lambda}$ is in the anthropic range (3), because that is where all the galaxies are.

\section{MULTIPLE BRANE NUCLEATION}

Up till now we assumed that brane nucleations change the four-form field $F$ by a single unit, Eq. (20). However, nucleation of multiple coincident branes is also possible. For $k$ coincident branes there is a $U(k)$ super Yang-Mills (SYM) living on the world volume. In FMSW [6] it was argued that the nucleation of coincident branes would be enhanced by a large degeneracy factor

$$
D=e^{S},
$$

where $S$ is the "entropy" of the SYM fields. For 2-branes arising from the wrapping of a 4D-brane on a degenerating 2-cycle, FMSW estimated this entropy as

$$
S \sim k^{\beta} R^{2} T^{2} .
$$

Here $k^{\beta}$ counts the effective number of degrees of freedom which live on the brane. There are theoretical uncertainties in the exponent $\beta$, but FMSW suggest that it should be between 2 and 3/2. $R$ is the radius of the Coleman-de Luccia instanton, which coincides with the size of the "bubble" at the time of nucleation, and $T$ is some effective temperature. FMSW considered two different candidates for the effective temperature. One of them was the effective ambient de Sitter temperature [32] $T_{0}$ before brane nucleation, and the other was the geometric mean of $T_{0}$ and the effective temperature $T_{I}$ of the new de Sitter space inside the nucleated brane $T$ $\sim\left(T_{I} T_{0}\right)^{1 / 2}$.

It is easy to understand, however, that the relevant effective temperature corresponding to the Coleman-de Luccia (CdL) instanton is in fact none of the above, but simply the effective de Sitter temperature of the $(2+1)$-dimensional world volume of the brane

$$
T=\frac{1}{2 \pi R}
$$

This is the temperature experienced by the degrees of freedom living in the wall (and it is in fact higher than $T_{0}$ and $T_{I}$ ). The prefactor $D$ is a determinant arising from Gaussian 
integration of perturbations around the instanton solution, including all light degrees of freedom. Such determinants where discussed in some detail in [23]. A scalar field of mass $m$ living on the $(2+1)$-dimensional world volume gives a contribution

$$
D_{s}=e^{\zeta^{\prime}(0) / 2}
$$

where $\zeta(z)$ is the Zeta function of the scalar fluctuation operator on the 3 -sphere. Its derivative at the origin is given by [23]

$$
\zeta^{\prime}(0)=2 \zeta_{R}^{\prime}(-2)-y^{2} \ln (\sin \pi y)+\frac{2}{\pi^{2}} \int_{0}^{\pi y} x \ln (\sin x) d x,
$$

where $y^{2}=1-m^{2} R^{2}$ and $\zeta_{R}$ is the usual Riemann Zeta function (this expression is valid for light fields, with $m R<1$ ).

For instance, the contribution of a conformally coupled scalar field can be obtained by taking $m^{2}=(3 / 4) R^{-2}$, which gives

$$
\zeta^{\prime}(0)=2 \zeta_{R}^{\prime}(-2)-\frac{1}{4} \ln 2+\frac{7}{8 \pi^{2}} \zeta_{R}(3) \approx-0.1276 .
$$

Hence, the effective degeneracy factor contributed by a conformal scalar field is given by

$$
D_{\text {conf. }} \approx e^{-.0638} \approx 0.91<1 .
$$

The first thing to note is that this factor is not an enhancement, but a suppression. Hence, the determinant cannot simply be thought of as the exponential of an entropy.

In fact, the CdL instanton is not a thermal instanton, but a zero temperature instanton. Thermal instantons for brane nucleation are static and have the topology $S^{2} \times S^{1}$ (rather than $S^{3}$ ), where the $S^{2}$ is the 2D-brane at fixed time and the $S^{1}$ is the periodic Euclidean time. Thermal instantons do in fact exist also in de Sitter space, but they have not received too much attention because their Euclidean action is always larger than that of the maximally symmetric CdL. For thermal instantons (in flat or in de Sitter space) the determinantal prefactor is given by $D=e^{-\Delta F / T}$, where $\Delta F=\Delta E-T S$ is the free energy contribution of light degrees of freedom on the brane. Such prefactors have been considered in [33]. The free energy consists of the vacuum energy $\Delta E$ (or Casimir energy on the two-sphere) minus the product of the temperature times the entropy. While the entropy is always positive, the sign of the Casimir contribution is notoriously dependent on the type of field. In fact, for thermal instantons in de Sitter the temperature is always smaller or equal to the inverse of the size of the bubble, and hence the sign of the free energy contribution can easily be dominated by the Casimir contribution, which can have either sign. Although as mentioned above the CdL instanton is not thermal, this consideration may help clarify why the prefactor $D$ need not represent an enhancement. Depending on the field content it may represent a suppression.

Another thing to note about Eq. (55) is that it is independent of $R$. Roughly speaking, this is consistent with the fact that the effective temperature is $T \propto R^{-1}$. In general, however, the degeneracy factor will depend on $R$ and on the mass of the particle. For light minimally coupled scalars, Eqs. (53) and (54) give

$$
D_{s} \approx \frac{e^{\zeta_{R}{ }^{\prime}(-2)}}{\pi^{1 / 2} m R} \quad(m R \ll 1)
$$

There can be a strong enhancement in the nucleation rate if there are very light massless scalar fields. In the limit $m$ $\rightarrow 0$ the factor goes to infinity. This is because a massless scalar has a normalizable zero mode on the sphere, corresponding to the symmetry $\phi \rightarrow \phi+$ const. In this case, the zero mode must be treated as a collective coordinate. The nucleation rate is proportional to the range $\delta \phi$ of the field $\phi$, because the bubbles can be nucleated with any average value of the scalar field with equal probability [23]

$$
\begin{aligned}
\delta D_{s}\left(m^{2}=0\right) & =\lim _{m^{2} \rightarrow 0}\left[m D_{s}(m)\right]\left(\pi R^{3}\right)^{1 / 2} \delta \phi \\
& =e^{\zeta_{R}{ }^{\prime}(-2)} R^{1 / 2} \delta \phi .
\end{aligned}
$$

As we shall discuss in some examples below, some scalars are likely to pick up masses of order of the intrinsic curvature of the $2+1$ sphere, and for these $D_{s}$ is also independent of the radius.

Let us briefly consider the field content on the brane. For $k$ coincident 4D-branes in ten dimensions, the effective theory is $U(k)$ super Yang-Mills (SYM) theory. This consists of a $U(k)$ gauge field plus $5\left(k^{2}-1\right)$ scalar degrees of freedom in the adjoint representation of $S U(k)$ plus 5 scalar singlets plus the corresponding fermionic degrees of freedom.

If the branes are flat (as in the case when there is no external four-form field), then the theory is supersymmetric and all scalar degrees of freedom are massless. For the case of a single brane, the five scalars represent the goldstone modes of the broken translational symmetry. That is, they correspond to transverse displacements of the brane. For the case of two branes, there are 10 such displacements. Five of them correspond to simultaneous motion of both branes. These are the singlets under $S U(2)$. The rest are in the adjoint representation, and if they acquire an expectation value they give mass to two of the four gauge bosons. For instance, when the two branes move apart, one of the adjoint scalars acquires an expectation value and two of the gauge bosons get a mass, breaking the symmetry $U(2) \rightarrow U(1) \times U(1)$.

The case of interest to us is not a flat brane, but a 4Dbrane wrapped on a degenerating two cycle. The world volume of the resulting 2D-brane in 4 Euclidean dimensions is not flat either, but forms a 3 -sphere of radius $R$. In this situation, we do not expect the theory to be supersymmetric. This is just as well, since in order for the instanton to make any sense, some of the adjoint scalars must pick up masses at one loop. Otherwise the instanton would have too many zero modes and too many negative modes. To simplify, let us consider the 3 -sphere in 4 non-compact dimensions. For the case of a single brane, the transverse displacements corre- 
spond to a scalar field of mass $m^{2}=-3 R^{-2}$ [34]. This scalar field has a single negative mode, which is the constant $l$ $=0$ mode. A negative mode is precisely what is needed for an instanton to contribute to the imaginary part of the vacuum energy, and hence to contribute to false vacuum decay [21]. Also, there are four normalizable zero modes, which are the spherical harmonics with $l=1$. These correspond to the four space-time translational modes of the instanton, which have to be treated as collective coordinates. This Goldstone field gives a determinantal prefactor of the form [23]

$$
D=\frac{\sigma^{2} R^{2}}{4} e^{\zeta_{R}^{\prime}(-2)} \Omega
$$

where $\Omega=V T$ is the spacetime volume. The prefactor $A$ in the nucleation rate (11) is obtained after dividing by $\Omega$.

If there are 2 coincident branes, then in principle there would be two such fields $\phi_{1}$ and $\phi_{2}$ corresponding to the independent transverse displacements of the brane. However, only the combination $\phi_{+}=\left(\phi_{1}+\phi_{2}\right)$ will correspond to a singlet under $S U(2)$. The orthogonal combination $\phi_{-}$ $=\left(\phi_{1}-\phi_{2}\right)$ will be in the adjoint. As mentioned above, if the instanton with two coincident branes is to make any sense, this combination must acquire a positive mass at one loop so that there is a single negative mode, not two, and four normalizable zero modes in total. In other words, in order for the instanton to make sense, the branes must attract each other. If they repelled each other or if they did not "interact," then the two brane configuration would in fact be an accidental superposition of two independent bubbles in the "dilute gas" of instantons. The mass of $\phi_{-}$can be estimated as follows. The mass of the gauge field $A_{\mu}$ is given by $m_{A}\left(\phi_{-}\right) \sim M_{P}^{2} f(d)$, where $M_{P}$ is the Planck mass, $d$ is the distance between branes and $f(d) \approx d$ for $d \gg M_{P}^{-1}$. This is because this vector corresponds to fundamental strings stretching from one brane to the other. For smaller distances, $d \leq M_{P}^{-1}$, we may expect a milder behavior for the mass, which we may heuristically parametrize as a power $f(d)$ $\approx d\left(M_{P} d\right)^{n}$, with $n>0$. The canonical field is related to the distance through $\phi_{-} \sim d \sigma^{1 / 2}$. Hence

$$
m_{A}^{2} \sim M_{P}^{4} f^{2}\left(\sigma^{-1 / 2} \phi_{-}\right) .
$$

On a flat brane, the effective potential induced by a gauge field of mass $m_{A}$ is proportional to $m_{A}^{3}$. However, it can be shown that on a sphere there is also a term of order $m_{A}^{2} R^{-1}$ which will in fact dominate at small $m_{A}$. When these terms are added to the tree level potential $-3 R^{-2} \phi_{-}^{2}$, the scalar acquires a very tiny expectation value $\left\langle\phi_{-}\right\rangle$ $\sim\left(\sigma / R M_{P}^{4}\right)^{1 / 2 n} \sigma^{1 / 2} M_{P}^{-1}$. In the broken phase, $\phi_{-}$has a positive mass squared of order $m_{\phi_{-}}^{2} \sim n R^{-2}$. The gauge bosons will in turn acquire imperceptibly tiny masses $m_{A}^{2}$ $\ll R^{-2}$.

To summarize, some of the scalars may get very large masses from the wrapping on a degenerating cycle. These will decouple, and presumably will not contribute to the degeneracy factor. Others, corresponding to the relative posi- tion of the 2-branes in 3+1 dimensions will get masses of order $R^{-1}$, and hence will contribute degeneracy factors of order one, just like the conformally coupled field discussed above. For the massless or nearly massless gauge fields the contribution to the degeneracy factor will be of order one (the vectors have no zero modes on the sphere, so unlike the case of a scalar, a tiny mass will not cause a large degeneracy factor). Similar considerations could be applied to fermions. Thus we expect the total prefactor to be of order

$$
D_{\text {total }} \sim \Omega \sigma^{2} R^{2} e^{a k^{\beta}}
$$

where $a$ is a numerical factor and $k^{\beta}$ is, as in Eq. (51), an estimate of the effective number of degrees of freedom. For a flat brane at weak coupling, $\beta=2$, but as argued by FMSW it could be lower for the wrapped brane. Unfortunately, without going into a very detailed analysis (which is outside the scope of this paper) we are unable to determine the sign of the constant $a$. However, as argued above, this value seems to be rather insensitive to the value of $R$ or to the value of the ambient de Sitter temperature.

For $a<0$ the nucleation of multiple branes is suppressed and we are back to the situation described in Sec. IV B. For $a>0$ a disaster may occur because transitions into deep anti-de Sitter space through multiple brane nucleation seem to be unsuppressed due to a large degeneracy factor. FMSW suggested that the disaster could be averted by an anthropic argument. If the step $\epsilon$ in the vacuum energy is of the order of 3 in units of $\rho_{M 0}$, allowing the values $\ldots,-2,1,4, \ldots$ then the stringent anthropic bound for a negative cosmological constant $\rho_{\Lambda} \geq-\rho_{M 0}$ would tell us that the vacuum energy is in fact the lowest allowed value in the list, that is $\rho_{\Lambda} \sim \rho_{M 0}$ (note that this argument requires a certain adjustment of the step $\epsilon$ in order to explain the observed value). However, there is another problem which is how to explain the stability of this vacuum once it has been reached. In the FMSW scenario, the stability was attributed to the fact that in the vacuum with $\rho_{\Lambda} \sim \rho_{M 0}$ the effective temperature of the brane would be so low that the degeneracy factor is switched off. However, as we have seen, the degeneracy factor is quite independent on the ambient de Sitter temperatures and hence it does not seem to switch off. The same mechanism that would enhance coincident brane nucleation from a high energy vacuum, would cause the disastrous decay of "our" vacuum.

Finally, we note that even if a mechanism could be devised to switch off the degeneracy factor, so that the present vacuum is stable, the time coincidence $t_{G} \sim t_{\Lambda}$ would be left unexplained by this approach (just as in the non-anthropic models discussed in Sec. VIII). Also, the unsuppressed nucleation of coincident branes seems to preclude eternal inflation, and even if one may intuitively argue that the lowest anthropic value is the most probable, the actual probability distribution for positive $\rho_{\Lambda}$ seems hard to estimate.

\section{SCALAR FIELD WITH A VERY FLAT POTENTIAL}

In this class of models, what we perceive as a cosmological constant is in fact a potential $V(\phi)$ of a scalar field $\phi(x)$. 
The potential does not have a succession of minima as in Abbott's washboard model, but its slope is assumed to be so small that the evolution of $\phi$ is slow on the cosmological time scale. This is achieved if the slow roll conditions

$$
\begin{aligned}
& M_{P}^{2} V^{\prime \prime} \ll V \lesssim \rho_{M 0}, \\
& M_{P} V^{\prime} \ll V \lesssim \rho_{M 0},
\end{aligned}
$$

are satisfied up to the present time (here it is assumed that any "true" cosmological constant is also included in the potential $V$.) These conditions ensure that the field is overdamped by the Hubble expansion, and that the kinetic energy is negligible compared with the potential energy (so that the equation of state is basically that of a cosmological constant term.) The field $\phi$ is also assumed to have negligible couplings to all fields other than gravity.

Let us now suppose, as in the previous sections, that there was a period of inflation driven by a different scalar field $\chi$. During inflation, massless scalar fields are randomized by quantum fluctuations, which cause their root mean squared value to increase with time as $\Delta \phi \sim H(H t)^{1 / 2}$, where $H$ is the inflationary expansion rate. If we consider a field of mass $m$, this effect competes with the classical drift down to the bottom of the potential, and after some time of order $t$ $\sim \mathrm{Hm}^{-2}$ a stationary distribution with root mean squared $\Delta \phi \sim H^{2} m^{-1}$ is established. This can be interpreted in terms of the Gibbons-Hawking temperature $T \sim H$ of de Sitter space as the condition $V(\phi) \sim m^{2} \phi^{2} \sim T^{4}$. In this example, all field values $|\phi| \ll H^{2} / m$ would be almost equally probable after the end of inflation. This discussion, however, assumes that inflation proceeds at almost the same rate for all field values in the range considered. That is, the differential expansion rate $\delta H \sim V(\phi)\left(H M_{P}^{2}\right)^{-1}$ is ignored.

The case of interest to us is slightly more general because the potential need not be quadratic, and also because we are not necessarily interested in field values near $\phi=0$. Rather, we are interested in field values for which the energy density is in the anthropically allowed range

$$
-\rho_{M 0} \lesssim V(\phi) \lesssim 100 \rho_{M 0} .
$$

The differential expansion rate $\delta H \sim V(\phi)\left(H M_{P}^{2}\right)^{-1}$ will be negligible if the time $t \sim(\Delta \phi)_{\text {anth }}^{2} H^{-3}$ that it takes for the field to fluctuate across the anthropic range of $\phi$ corresponding to Eq. (59) is smaller than $(\delta H)^{-1}$ for the same range. This requires

$$
(\Delta \phi)_{a n t h} \ll \frac{H^{2}}{10 \rho_{M 0}^{1 / 2}} M_{P} .
$$

If this condition is not satisfied, then the a priori probability for the field values with a higher $V(\phi)$ would be exponentially enhanced with respect to the field values at the lower anthropic end. This would result in a prediction for the effective cosmological constant which would be too high compared with observations. Therefore, in what follows, we shall demand that our potential satisfies Eqs. (57), (58) and (60).

\section{A. Solving both cosmological constant problems}

Consider a potential of the form [4]

$$
V(\phi)=\rho_{\text {bare }}+\frac{1}{2} \mu^{2} \phi^{2},
$$

where $\rho_{\text {bare }}$ represents the "true" cosmological constant. If $\rho_{\text {bare }}$ and $\mu^{2}$ have opposite signs, then the effective vacuum energy will be very small when

$$
|\phi| \approx\left|2 \rho_{\text {bare }}\right|^{1 / 2}|\mu|^{-1} .
$$

The anthropic range is given by $(\Delta \phi)_{\text {anth }}$ $\sim 100 \rho_{M 0}\left|\mu^{2} \rho_{\text {bare }}\right|^{-1 / 2}$. Then, conditions (57), (58) and (60) imply

$$
10^{3} \frac{\rho_{M 0}^{1 / 2}}{H^{2}} \frac{\rho_{M 0}}{\left|\rho_{\text {bare }}\right|^{1 / 2} M_{P}} \ll|\mu| \ll \frac{\rho_{M 0}}{\left|\rho_{\text {bare }}\right|^{1 / 2} M_{P}} .
$$

From the cosmic microwave background temperature fluctuations we know that $H \leqslant 10^{-5} M_{P}$. This leaves a wide range of possibilities for the value of the mass parameter,

$$
\mu \sim\left(10^{-167}-10^{-120}\right) \frac{M_{P}^{3}}{\left|\rho_{\text {bare }}\right|^{1 / 2}},
$$

spanning some 47 orders of magnitude. Provided that $\mu$ is in this range, the a priori probability distribution $\mathcal{P}_{*}(\phi)$ for $\phi$ will be flat. The probability distribution for the effective cosmological constant $\rho_{\phi}=V(\phi)$ is given by

$$
\mathcal{P}_{*}\left(\rho_{\phi}\right)=\frac{1}{V^{\prime}} \mathcal{P}_{*}(\phi),
$$

and it will also be very flat, since $V^{\prime}$ is almost constant in the anthropic range. As mentioned in Sec. II, a flat a priori distribution for the effective cosmological constant in the anthropic range entails an automatic explanation for the two cosmological constant puzzles $[16,17]$.

\section{B. A small mass from instantons?}

The challenge in the scenario presented above is to explain the small mass parameter (64). In Ref. [4] we suggested that this can be achieved through instanton effects. For instance, $\phi$ could be a pseudo Goldstone boson, the phase of a scalar field $\Phi=\eta e^{i \phi / \eta}$ which spontaneously breaks a global $U(1)$ symmetry. Since global charge can be swallowed by wormholes, a small mass term for the field $\phi$ will be generated through gravitational instantons $[35,36]$.

Another possibility is that the phase may have an "axion" coupling of the form

$$
\frac{\alpha_{s}}{\eta} \phi \widetilde{F} F,
$$

where $F$ is the field strength of a "hidden" gauge sector with gauge coupling constant $\alpha_{s}$. The coupling (65) will give a small mass to the pseudoscalar $\phi$ through instanton effects. 
We should mention, however, that there may be certain limitations in implementing this idea in the present context. Consider an instanton-induced potential of the form

$$
V(\phi)=\rho_{\text {bare }}+\Lambda^{4} \cos (\phi / \eta) .
$$

In order to solve the cosmological constant problem we need

$$
\Lambda^{4} \gtrsim \rho_{\text {bare }} .
$$

Combining this with the slow roll conditions (57) and (58) we find

$$
\eta \gg M_{P} \frac{\rho_{\text {bare }}}{\rho_{M 0}} .
$$

Thus, the expectation value $\eta$ must be truly huge compared with the Planck scale.

In usual axion models, the effective vertex (65) can be obtained in the following way. The scalar field $\Phi$ has Yukawa interactions of the form $h \Phi \bar{\Psi} \Psi$ with an "exotic" fermion $\Psi$ (here $h$ is the Yukawa coupling constant). The fermion in turn interacts with the non-Abelian gauge fields, and the coupling (65) is generated at one loop. The mass of the fermions in the broken phase is given by $m_{\Psi} \sim h \eta$. In our case, this mass is extremely large (unless $h$ is extremely small), and so we can hardly trust the field theory model for generating Eq. (65).

Perhaps more worrisome is the effect of wormholes. For small symmetry breaking scale $\eta \leqslant M_{P}$ the scale $\Lambda^{4}$ in the instanton potential is of order $M_{P}^{4} e^{-S}$, where $S \sim M_{P} / \eta$ is the wormhole action $[35,36]$. The radius of the wormhole is given by $R \sim\left(M_{P} \eta\right)^{-1 / 2}$. This radius approaches the Planck scale as $\eta$ approaches $M_{P}$, and the process becomes unsuppressed. The instanton calculation becomes unreliable for higher values of $\eta$, but it is not clear what would prevent nonperturbative gravitational effects from completely destroying the global symmetry.

Therefore, as mentioned above, the generation of a small mass through instantons may not have a straightforward implementation in the present context. Clearly, this issue deserves further investigation (see e.g. [36]).

\section{A very flat potential from field renormalization}

Consider a potential of the form [18]

$$
V(\phi)=\rho_{\text {bare }}+M^{4} f(\lambda \phi),
$$

where $M$ is a reasonable mass parameter and $f$ is a function of order one with no large or small parameters. If the parameter $\lambda$ in the argument of $f$ is chosen to be very small, then $V(\phi)$ will be very flat. In particular, the mass term of the field $\phi$ which has two powers of $\lambda$ will be very small. Weinberg suggested [18] that perhaps the smallness of $\lambda$ can be attributed to a large running of the field renormalization $Z_{\mu}$ from some fiducial short distance scale $\mu$ to the large scales in which the cosmological constant is relevant, $\mu \rightarrow 0$.
More generally, as noted in Ref. [9], the effective Lagrangian for a scalar field $\psi$ at large distances will include nonminimal kinetic terms:

$$
\mathcal{L}=F^{2}(\psi)\left(\partial_{\mu} \psi\right)^{2}-V(\psi)+\cdots
$$

Here $F$ plays the role of a field renormalization, which in fact may depend on $\psi$, and we have omitted terms with more derivatives of $\psi$. If $F$ is very large, then the field redefinition

$$
d \phi=F d \psi
$$

will result in a very flat effective potential for $\phi$.

Take for instance $F=e^{\psi / M_{P}}$ and $V=\rho_{\text {bare }}+\left(m^{2} / 2\right) \psi^{2}$, where $m$ is a not too large mass parameter (see below). After the change of variables we obtain

$$
\mathcal{L}=\left(\partial_{\mu} \phi\right)^{2}-\rho_{\text {bare }}-\frac{1}{2} m^{2} M_{P}^{2}\left[\ln \left(\phi / M_{P}\right)\right]^{2}+\cdots .
$$

The effective potential is now very flat at large $\phi$. The slow roll conditions (57) and (58) are satisfied for

$$
\phi \gtrsim \phi_{\min }=M_{P} \frac{m^{2}}{H_{0}^{2}} \ln \frac{m}{H_{0}} .
$$

The antropic range $m^{2} \psi^{2} \sim\left|\rho_{\text {bare }}\right|$ will satisfy this condition provided that $m \ll\left|\rho_{\text {bare }}\right|^{1 / 2} M_{P}^{-1}$. Finally, the condition (60) is easily satisfied by choosing a sufficiently high Hubble rate during inflation $H^{2} \gg 10^{3} \rho_{0 M}^{1 / 2}\left(\phi / \phi_{\min }\right)$.

Thus, starting from a Lagrangian (67) with fairly simple functions $F$ and $V$ we have been able to satisfy all necessary conditions to solve both cosmological constant problems. Of course, one may wonder why $F$ should have exponential behavior when $V$ is only polynomial, and it would be good to find a well motivated physical setup where this Lagrangian emerges in a natural way.

\section{A SLOWLY VARYING FOUR-FORM?}

In theories with extra dimensions, the four form field strength is dynamical above the compactification energy scale. Donoghue suggested [9] that in the early universe the four-form might take a continuum of different values in different parts of the universe, and that it might get frozen to these values as the universe cooled down below the compactification scale. However, it is easy to show that the effective cosmological constant can vary from place to place only if the size of the internal space is also variable. As a result, the effect of the four form is more properly described as a contribution to the effective potential for the radius modulus of the extra space.

Let the higher dimensional manifold be the product of a four-dimensional spacetime $\mathcal{M}$ and an internal space $\mathcal{S}$,

$$
d s^{2}=g_{\mu \nu}(x) d x^{\mu} d x^{\nu}+\sigma_{i j}(x, y) d y^{i} d y^{j},
$$

where $\mu, \nu=0, \ldots, 3$ are the four-dimensional indices and $i, j=1, \ldots, n$ are the internal space indices. The field strength takes the form, $\mathbf{F}=f(x, y) \omega_{4}+\ldots$, where $\omega_{4}=\sqrt{g}$ 
$\left(\wedge_{\mu} d x^{\mu}\right)$ is the four-dimensional volume form ( $g=$ $\left.-\operatorname{det} g_{\mu \nu}\right)$ and the ellipsis denote terms with at least one internal index (these do not behave as a four-form upon dimensional reduction). The equations of motion reduce to

$$
d \mathbf{G}=0
$$

where $\mathbf{G}=* \mathbf{F}=f(x, y) \omega_{\mathbf{n}}+\cdots$ is the dual of the field strength and $\omega_{\mathbf{n}}=\sqrt{\sigma}\left(\wedge_{i} d y^{i}\right)$ is the volume form on the internal space $\left(\sigma=\operatorname{det} \sigma_{i j}\right)$. Consider two different points $x_{1}$ and $x_{2}$ on the 4D manifold $\mathcal{M}$, and a curve $\gamma_{12}$ joining them. Applying Stokes theorem to the "cylinder" $\gamma_{12} \times \mathcal{S}$ (where $\mathcal{S}$ is the internal space), and using the equations of motion (69), we immediately find that

$$
\int_{S ; x_{1}} \mathbf{G}-\int_{S ; x_{2}} \mathbf{G}=\int_{\mathcal{S} \times \gamma_{12}} d \mathbf{G}=0
$$

for arbitrary $x_{1}$ and $x_{2}$. Assume that the internal metric factorizes as

$$
\sigma_{i j}=e^{2 \psi(x)} \tilde{\sigma}_{i j}(y) .
$$

Then, Eq. (70) implies

$$
\bar{f}(x)=f_{0} e^{-n \psi(x)},
$$

where $\bar{f}(x)$ is the average of $f(x, y)$ over the internal space and $f_{0}$ is a constant. Kaluza-Klein modes average to zero on the internal space and do not contribute to $\bar{f}$. However such modes are massive in the reduced theory and do not behave as an effective cosmological constant. It follows that the contribution of the four form to the effective cosmological constant is

$$
\frac{1}{2} F^{2} \equiv \frac{1}{2 \times 4 !} \int_{\mathcal{S}} F_{\mu \nu \rho \sigma} F^{\mu \nu \rho \sigma} \sqrt{\sigma} d^{n} y=\frac{1}{2} F_{0}^{2} e^{-n \psi(x)},
$$

where $F_{0}^{2}=f_{0}^{2} \int_{\mathcal{S}} \sqrt{\tilde{\sigma}} d^{n} y=$ const.

In the dimensionally reduced theory, $\psi(x)$ is a four dimensional scalar field, and Eq. (71) is just a contribution to its effective potential $V(\psi)$. At the classical level, there are two other such contributions, due to a bare higher dimensional cosmological constant $\Lambda_{\text {bare }}^{(4+n)}$ and due to the curvature of the internal manifold. Following Refs. [37], it is easy to show that in terms of the Einstein frame metric $\bar{g}_{\mu \nu}$ $=e^{n \psi} g_{\mu \nu}$, the effective action takes the form

$$
S=\frac{M_{P}^{2}}{2} \int \sqrt{\bar{g}} d^{4} x\left[\bar{R}-\frac{n(2+n)}{2} \bar{g} \mu \nu \partial_{\mu} \psi \partial_{\nu} \psi-V(\psi)\right],
$$

with

$$
V(\psi)=\Lambda_{0} e^{-n \psi}+\frac{F_{0}^{2}}{2} e^{-3 n \psi}-\frac{K}{2} e^{-(2+n) \psi} .
$$

Here $M_{P}^{2}=M_{*}^{2+n} V_{0}$ and $\Lambda_{0}=\Lambda_{\text {bare }}^{(4+n)} V_{0}$, where $M_{*}$ is the higher dimensional Planck mass, $V_{0}=\int_{\mathcal{S}} \sqrt{\tilde{\sigma}} d^{n} y=$ const., and $K$ is defined by $\widetilde{R}_{i j}=(K / n) \widetilde{\sigma}_{i j}$. In general, the potential may have a minimum but this need not be near $V=0$. For $n>1$ one can adjust the parameters $\Lambda_{0}$ and $F_{0}$ so that the minimum is at the right height to fit observations, but this would be the usual fine-tuning (for $n=1$ the minimum must have negative effective cosmological constant, so this tuning is not possible). We may also consider the possibility that the field $\psi$ is away from the minimum, but slowly rolling so that the effective potential $V(\psi)$ plays the role of an effective cosmological constant, as described in Sec. VI. The problem is that if the slow roll conditions are met, then $\psi$ would have a negligible mass and would mediate long range interactions of gravitational strength, which are ruled out by observations.

\section{NON-ANTHROPIC APPROACHES}

Here we comment on some attempts to solve the cosmic coincidence problem without resorting to the anthropic principle. In a recent paper [7] Arkani-Hamed et al. suggested an explanation to the approximate coincidence of several cosmological timescales: the time of matter-radiation equality $t_{e q}$, the time of $\Lambda$-domination $t_{\Lambda}$, and the time of galaxy formation $t_{G}$. They assume that the Planck scale $M_{P}$ and the electroweak scale $M_{w}$ are the only relevant scales and argue that the temperature at matter-radiation equality and the vacuum energy should then be given by

$$
\begin{aligned}
& T_{e q} \sim M_{w}^{2} / M_{P}, \\
& \rho_{\Lambda} \sim\left(M_{w}^{2} / M_{P}\right)^{4} .
\end{aligned}
$$

It follows immediately from Eqs. (72),(73) that $t_{e q} \sim t_{\Lambda}$. This coincidence should of course be understood in a very rough sense, since the actual values of $t_{e q}$ and $t_{\Lambda}$ in our universe differ by a few orders of magnitude. Now, assuming the density fluctuation amplitude

$$
Q \equiv \delta \rho / \rho \sim 10^{-5},
$$

and using a more accurate value for $t_{e q} / t_{\Lambda}$, the authors show from Eq. (73) that the epoch of galaxy formation is at

$$
t_{G} \sim t_{\Lambda}
$$

In our view, a relation like Eq. (73) may account for the smallness of $\Lambda$ and may even explain its observed value. However, the cosmic coincidence (75) would remain unexplained. The time of $\Lambda$-domination is determined by the value of $\Lambda$, while the epoch of galaxy formation is determined by the amplitude of density fluctuations $Q$. Even if we explain the value of $\Lambda$, we still have to explain why the value of $Q$ is such that $t_{G} \sim t_{\Lambda}$. Moreover, the accuracy of a few orders of magnitude is not sufficient to explain the cosmic time coincidence: observations indicate that the coincidence (75) is accurate within one order of magnitude.

Another non-anthropic approach to solving the cosmic coincidence problem involves $k$-essence, a scalar field with a non-trivial kinetic term [8]. $k$-essence has a positive effective pressure during the radiation era and starts acting as an ef- 
fective cosmological constant with the onset of matter domination. With a suitable choice of parameters it dominates the universe at $t_{\Lambda} \sim t_{G}$. However, one could also choose parameters to obtain $t_{\Lambda} \ll t_{G}$ or $t_{\Lambda} \gg t_{G}$. This model can explain why $\Lambda$-domination occurs at $t>t_{e q}$, but it cannot account for the coincidence (75).

\section{MODELS WITH VARIABLE Q}

Several authors have recently expressed the view that the anthropic principle can perhaps be applied to the cosmological constant problem-but to nothing else $[38,26]$. For instance, Weinberg has remarked [18] that we cannot explain the masses and charges of the elementary particles by assuming that they depend on the expectation values of scalar fields with very flat potentials. These light fields would couple to the elementary particles, and would have been observed in collisions and decays.

While this remark may be true, we can still apply the anthropic principle to variables which determine the large scale properties of the Universe, and which generically fall into the category of "initial conditions." Examples of these are the amplitude of primordial fluctuations $Q[41,39,16]$, the density parameter $\Omega[40,39]$, or even the baryon asymmetry. In the inflationary context, these parameters depend on the path that the inflaton field takes in going from the diffusion regime to thermalization. The inflaton potential represented in Fig. 2 is one-dimensional, and there is a single path from the top of the potential to the local minimum. However, more generally, the inflaton has several components, and there may be a continuum of paths from the diffusion region to a given minimum. Even if the low energy particle physics Lagrangian is the same in all thermalized regions, and even if there are no exotic light degrees of freedom after thermalization, these regions may start with different initial conditions which will be more or less favorable to galaxy formation.

Consider for instance [39] a two component scalar field $\chi=\chi_{1}+i \chi_{2}=|\chi| e^{i \Theta}$, with potential $V(\chi)=\left(g_{1} \chi_{1}^{2}+g_{2} \chi_{2}^{2}\right) / 2$. This potential produces inflation for $|\chi| \gtrsim M_{P}$. However, the amplitude of density perturbations $Q$ depends on the direction $\Theta$ of approach to the minimum, $Q \sim m(\Theta) N(|\chi|) M_{P}^{-1}$. Here $m^{2}(\Theta)=g_{1} \cos ^{2} \Theta+g_{2} \sin ^{2} \Theta$ and $N \sim|\chi|^{2} M_{P}^{-2} \sim 60$ is the number of e-foldings from the time the present Hubble scale first crossed the horizon until the end of inflation. The minimum at $\chi=0$ will be reached from different directions in different thermalized regions, and therefore these regions will have a different value of $Q$ as an initial condition. This example illustrates that $Q$ can easily be made into a random variable. In general, its a priori distribution $\mathcal{P}_{*}(Q)$ (i.e. its volume distribution at the time of thermalization) will not necessarily be flat in the anthropically allowed range. For any given model, this distribution can be calculated using the numerical methods of Ref. [29]. To proceed, however, we shall heuristically parametrize it as

$$
d \mathcal{P}_{*}(Q) \sim Q^{-\alpha} d \ln Q,
$$

where $\alpha$ is a constant (this may not necessarily be a good estimate for the particular model presented above, but we shall use it anyway for the sake of argument).

We may now take a point of view which is "complementary" to the one used in the preceeding sections. Let us assume that the cosmological constant is truly a constant of order $M_{w}^{8} M_{P}^{-6}$ determined from the fundamental theory (as assumed e.g. in [7]), and that $Q$ is a random variable with prior distribution (76). If $\alpha>0$, then low values of $Q$ will be favored a priori. However, if $Q$ is too low, galaxies will not have time to form before the time $t_{\Lambda}$ when the cosmological constant starts dominating. With this, we would basically explain why $Q \sim 10^{-5}$ as well as the time coincidence $t_{G}$ $\sim t_{\Lambda}$. These arguments can be made more quantitative in the following way. The probability distribution for a galaxy to form at time $t_{G}$ is given by

$$
d \mathcal{P}\left(t_{G}\right) \propto \mathcal{P}_{*}(Q) \frac{d \nu\left(t_{G}, t_{\Lambda}, Q\right)}{d t_{G}} d \ln Q d t_{G} .
$$

Here, $\nu\left(t_{G}, t_{\Lambda}, Q\right)$ is the fraction of matter that clusters up to the time $t_{G}$ in a universe where the density contrast at the time of recombination is $Q$ and where the cosmological constant is such that it will start dominating at the time $t_{\Lambda}$. This fraction can be easily estimated by using the Press-Schechter approximation. In Ref. [16] it was shown [see Eq. (27) of that reference] that after integrating over $Q$ the probability distribution for $t_{G}$ is given by

$$
d \mathcal{P}\left(t_{G}\right) \propto \frac{d F^{\alpha}}{d x} d x
$$

where

$$
F(x)=\frac{5}{6}\left(\frac{1+x}{x}\right)^{1 / 2} \int_{0}^{x} \frac{d w}{w^{1 / 6}(1+w)^{3 / 2}},
$$

and

$$
x=\sinh ^{2}\left(t_{G} / t_{\Lambda}\right) .
$$

(Following [16], we are using the convention that $t_{\Lambda}$ is the time at which $\Omega_{\Lambda}=\Omega_{M} \sinh ^{2} 1$, where $\Omega_{i}$ are the fractional densities of cosmological constant and non-relativistic matter respectively.) The distribution (78) is plotted in Fig. 3 for different values of $\alpha$, and we see that for moderate values of $\alpha$ it presents a rather prominent peak at $t_{G} \sim t_{\Lambda}$, as expected from the general arguments above.

Finally, one may take the view that both $\Lambda$ and $Q$ are random variables. This possibility was considered in [16], where it was shown that a decreasing a priori distribution for $Q$ pushes the cosmological constant to small values, so that both $t_{G}$ and $t_{\Lambda}$ tend to be very large. In this case, a new time scale comes into play. This is the so-called cooling boundary $t_{c b}$ [41]. For times $t>t_{c b}$ gravitationally collapsing clouds of galactic mass cannot fragment into stars because they are too cold to reach the usual "cooling" line emission thresholds, and they stay as pressure supported configurations for a very long time. Thus, usual galaxy formation is suppressed after $t \sim t_{c b} \approx 3 \times 10^{10} \mathrm{yr}$. This time is determined from micro- 


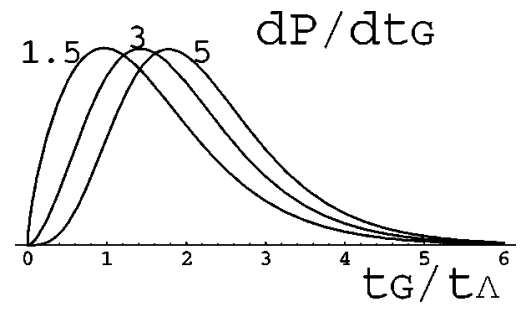

FIG. 3. Probability distribution for $t_{G} / t_{\Lambda}$, the time of galaxy formation as compared to the time when the cosmological constant starts dominating. Here $\Lambda$ is taken to be a fundamental constant but the density contrast $Q$ is treated as a random variable with a priori volume distribution $\propto Q^{-\alpha}$ at the time of thermalization. The plot is shown for $\alpha=1.5,3$ and 5. The distributions present rather well defined peaks at $t_{G} \sim t_{\Lambda}$.

physical parameters such as the fine structure constant, the proton mass and the fraction of baryonic matter. Since the time of galaxy formation cannot be arbitrarily large, in the situation where both $Q$ and $\Lambda$ are random variables we expect $t_{G} \sim t_{\Lambda} \sim t_{c b}$ (see [16] for details). There are many uncertainties associated with the calculation of $t_{c b}$. Perhaps after some of these uncertainties are removed, we may actually find that $t_{c b} \gg t_{G} \sim t_{\Lambda}$. This hypothetical situation would suggest that one of the time scales $t_{G}$ and $t_{\Lambda}$ is not a random variable, or that if both of them are, then their a priori distribution must have a rather peculiar behavior. This in turn would give us information on the theories of initial conditions giving rise to these a priori distributions.

These examples seem to suggest that the applicability of anthropic reasoning, once it is accepted, may easily go beyond the issue of the cosmological constant problem.

\section{CONCLUSIONS}

The anthropic principle has a bad reputation. It is often regarded as a handwaving argument relying on poorly understood phenomena like intelligent life and having no predictive power. Although this criticism is not entirely ungrounded, there is a class of cosmological models where the use of anthropic principle is not only justified but may in fact be inevitable, and where it can be used to make quantitative predictions. These are the models in which some cosmological parameters, or physical "constants," take different values in different parts of the universe. In such models, one cannot predict the precise values of the parameters that we are going to observe. One can only hope to calculate the corresponding probability distributions. The criteria for justifying (and compelling) the use of anthropic principle are that the model should provide (i) a mechanism for variation of the parameters and (ii) a way of calculating the probability distributions. Once the probabilities are calculated, one can predict that the parameters are going to be observed within a certain range of values, say, at a $95 \%$ confidence level. This seems to be as quantitative as one can possibly get in this class of models.

From a practical point of view, parameters that we can hope to determine anthropically should satisfy the condition that they do not affect life processes, and preferably should also not affect the poorly understood astrophysical processes such as star formation $[40,16,26]$. So for example the gravitational constant may be hard to determine anthropically with our present level of understanding, since it affects both the evolution of life and star formation. However, gravity does not change chemistry, which is already a big simplification. So it is not inconceivable that the value of Newton's constant may in the future receive an anthropic explanation.

In this paper we discussed anthropic approaches to solving the two cosmological constant problems (CCPs). The first (old) $\mathrm{CCP}$ is the discrepancy between the observed small value of $\rho_{\Lambda}$ and the large values suggested by particle physics models. The second (time coincidence) CCP is the puzzling coincidence between the epoch of galaxy formation $t_{G}$ and the epoch of $\Lambda$-domination $t_{\Lambda}$. While it is conceivable that the old CCP can be resolved by fundamental physics $[42,43,7,44]$, we have argued that the time coincidence problem calls for an anthropic explanation.

We first considered models with a discrete spectrum of $\rho_{\Lambda}$. These include Abbott's scalar field model with a "washboard" potential [19], as well as models in which $\rho_{\Lambda}$ can change through brane nucleation accompanied by a change of the four-form field $F[25,5,6,9]$. Such models can solve both CCPs, provided that (i) the separation between the discrete values of $\rho_{\Lambda}$ is $\epsilon \lessgtr \rho_{M 0}$, where $\rho_{M 0}$ is the present matter density, (ii) the probability distribution for $\rho_{\Lambda}$ at the end of inflation is nearly flat, $\mathcal{P}_{*}\left(\rho_{\Lambda}\right) \approx$ const, and (iii) the brane nucleation rate is sufficiently low, so that the present vacuum energy does not drop significantly in less than a Hubble time. We discussed the cosmology of this class of models, the calculation of the prior distribution $\mathcal{P}_{*}\left(\rho_{\Lambda}\right)$, and the observational constraints on the model parameters.

The required values of the "level separation" $\epsilon$ may appear uncomfortably small, but Feng, March-Russell, Sethi and Wilczek (FMSW) [6] have argued that they can naturally arise due to non-perturbative effects in $\mathrm{M}$ theory. In M-theory-related models, the brane tension $\sigma$ is related to $\epsilon$ through $\epsilon \sim \sigma \rho_{\text {bare }}^{1 / 2} / M_{p}$ and should also be very small. Our analysis shows that in such models the conditions (i)-(iii) cannot be satisfied without fine-tuning of the parameters.

It was conjectured by Weinberg [12] that the condition (ii) of a flat a priori distribution for $\Lambda$ would automatically be satisfied in any particle physics model where the cosmological constant is a random variable. In Ref. [4] we showed that this conjecture is not always satisfied in models where the role of the cosmological constant is played by a slowly varying field. Here, we have shown that the conjecture is generically not satisfied in four-form models either. In fact, this condition has to be enforced in order to fit observations. This, in turn, places severe constraints on the model parameters. Hence, in trying to solve the cosmological constant problems by anthropic means, the flat a priori distribution for $\Lambda$ cannot be taken for granted and the problem of calculating $\mathcal{P}_{*}$ has to be addressed.

Bousso and Polchinsky [5] have studied models with multiple four-form fields $F_{i}$ and found that the spectrum of the allowed values of $\rho_{\Lambda}$ can be sufficiently dense even for large brane tensions. However, in this case the vacua with nearby values of $\rho_{\Lambda}$ have very different values of $F_{i}$, and a flat 
probability distribution required in (ii) is rather unlikely. Moreover, the low-energy physics in such vacua is likely to be different, and it appears that the anthropic approach to solving the CCPs cannot be applied in this case [26].

For models unrelated to M-theory, $\sigma$ and $\epsilon$ are generally unrelated, and values consistent with the constraints (i)-(iii) can easily be found. However, if one gives up the M-theory connection, then the FMSW argument cannot be used, and one has to seek an alternative explanation for the tiny value of $\epsilon$. Alternatively, one might seek modifications of the FMSW model that could relax the relation between $\epsilon$ and $\sigma$.

All of the earlier discussions of the cosmology of discrete $\Lambda$ models encountered the "empty universe problem" $[31,19,25,5,6,9,26]$. In order to make the present vacuum sufficiently stable, the brane nucleation has to be strongly suppressed. One then finds that the time it takes the universe to evolve from some initial high value of $\rho_{\Lambda}$ to the present low value is much greater than the present Hubble time. This suggests that by the time the process is complete, any matter that the universe initially had may get diluted to an extremely low density, so that one would end up with an empty universe dominated by the cosmological constant.

We have argued that the empty universe problem disappears when the eternal nature of inflation is taken into account. During inflation, brane nucleations leading to higher and lower values of $\rho_{\Lambda}$ have nearly equal probabilities. As a result, the values of $\rho_{\Lambda}$ are randomized, with different parts of the universe thermalizing with different values. The resulting probability distribution $\mathcal{P}_{*}\left(\rho_{\Lambda}\right)$ can be calculated using the stochastic formalism we developed in Sec. IV. The slow rate of brane nucleation is not a problem in eternal inflation, since an unlimited amount of time is available.

FMSW suggested an interesting possibility that nucleation of multiple branes could be enhanced by a large degeneracy factor due to the light fields living on the branes. If true, this could significantly modify the brane model cosmology. In Sec. V we studied multiple brane nucleation in some detail and found that the pre-exponential factor in the brane nucleation rate can both enhance and suppress multiple brane nucleation, depending on the field content of the branes. We also concluded that models in which multiple brane nucleation dominates can be ruled out, because in such models there is nothing to prevent our present vacuum from tunneling down to deep anti-de Sitter space.

We also discussed models with a continuous spectrum of $\rho_{\Lambda}$, in which the role of the cosmological constant is played by the potential $V(\phi)$ of a scalar field $\phi(x)$. The potential has to be very flat, so that its value does not significantly evolve on the present Hubble time scale. The values of the field $\phi$ are randomized by quantum fluctuations during inflation, and models can easily be constructed in which the resulting probability distribution for $V(\phi)$ is nearly flat in the range of interest, thus solving both CCPs. The challenge here is to justify the very flat potentials required in this class of models. Possibilities include a pseudo-Goldstone field which acquires a potential through instanton effects [4], a large running of the field renormalization [18], and a non-minimal kinetic term with an exponential $\phi$-dependence [9]. We have pointed out some difficulties of the instanton approach.

We thus see that both discrete and continuous $\Lambda$ models could in principle solve both of the CCPs. However, none of the models that have been suggested so far appears particularly well motivated or natural.

An alternative approach is to assume that the old CCP can be solved within the fundamental theory. The cosmological constant is then truly a constant and is given by an expression such as $\rho_{\Lambda} \sim M_{w}^{4} M_{P}^{-2}$, as in [7]. At the same time, the amplitude of density fluctuations $Q$ could be a random variable, so that the epoch of galaxy formation $t_{G}$ is different in different parts of the universe. We have shown in Sec. VIII that, for a wide class of prior distributions $\mathcal{P}_{*}(Q)$, most of the galaxies will be in regions where $t_{G} \sim t_{\Lambda}$, thus explaining the cosmic time coincidence. It would be interesting to extend this analysis and calculate the distribution $\mathcal{P}_{*}(Q)$ for some models with a variable $Q$. One would then have some idea of how naturally the distributions of the required type can be obtained.

\section{Notes added}

After this paper was submitted for publication, a revised version of Ref. [6] has appeared. There, it is pointed out that the relation (21) between the tension $\sigma$ and the charge density $q$ of the brane does not hold for branes wrapping on degenerating cycles. Instead, the tension is suppressed by an exponential factor relative to the charge. We note two potential problems with this picture. First, as it was argued in Ref. [45], the brane charge and tension appear to be unprotected against renormalization below the supersymmetry breaking scale. Such renormalization would make the brane charge $q$ unacceptably large. Second, if for some reason the brane parameters do not get renormalized, then, in order to satisfy the anthropic constraint (22) on $q$, the brane tension has to be exceedingly small. The instanton action (9) would then be small and brane nucleation would be completely unsuppressed.

A new approach to explaining very flat scalar potentials and branes with a very small four-form charge has been suggested in Ref. [45], where these features are attributed to a spontaneously broken discrete symmetry.

\section{ACKNOWLEDGMENTS}

J.G. is grateful to Alex Pomarol and to Klaus Kirsten for useful and enjoyable discussions. This work was supported by the Templeton Foundation under grant COS 253. J.G. is partially supported by CICYT, under grant AEN99-0766, and by the Yamada Foundation. A.V. is partially supported by the National Science Foundation. 
[1] S. Perlmutter et al., Astrophys. J. 483, 565 (1997); S. Perlmutter et al., Bull. Am. Astron. Soc. 29, 1351 (1997); B. Schmidt et al., Astrophys. J. 507, 46 (1998); A. J. Riess et al., Astron. J. 116, 1009 (1998).

[2] A. Vilenkin, Phys. Rev. Lett. 74, 846 (1995).

[3] G. Efstathiou, Mon. Not. R. Astron. Soc. 274, L73 (1995).

[4] J. Garriga and A. Vilenkin, Phys. Rev. D 61, 083502 (2000).

[5] R. Bousso and J. Polchinski, J. High Energy Phys. 06, 006 (2000).

[6] J. L. Feng, J. March-Russell, S. Sethi, and F. Wilczek, Nucl. Phys. B602, 307 (2001).

[7] N. Arkani-Hamed, L. J. Hall, C. Kolda, and H. Murayama, Phys. Rev. Lett. 85, 4434 (2000).

[8] C. Armendáriz-Picon, P. Steinhardt, and V. F. Mukhanov, Phys. Rev. Lett. 85, 4438 (2000); Phys. Rev. D 63, 103510 (2001).

[9] J. Donoghue, J. High Energy Phys. 08, 022 (2000).

[10] J. D. Barrow and F. J. Tipler, The Anthropic Cosmological Principle (Clarendon, Oxford, 1986), p. 414.

[11] For an early attempt to apply anthropic arguments to the cosmological constant, see also P. C. W. Davies and S. Unwin, Proc. R. Soc. London 377, 147 (1981).

[12] S. Weinberg, Phys. Rev. Lett. 59, 2607 (1987).

[13] W. H. Press and P. Schechter, Astrophys. J. 187, 425 (1974).

[14] H. Martel, P. R. Shapiro, and S. Weinberg, Astrophys. J. 492, 29 (1998).

[15] S. Weinberg, in Critical Dialogues in Cosmology, edited by N. G. Turok (World Scientific, Singapore, 1997).

[16] J. Garriga, M. Livio, and A. Vilenkin, Phys. Rev. D 61, 023503 (2000).

[17] S. Bludman, Nucl. Phys. A663-664, 865 (2000).

[18] S. Weinberg, Phys. Rev. D 61, 103505 (2000); talk given at 4th International Symposium on Sources and Detection of Dark Matter in the Universe (DM 2000), Marina del Rey, California, astro-ph/0005265.

[19] L. Abbot, Phys. Lett. B 195, 177 (1987).

[20] S. Coleman and F. De Luccia, Phys. Rev. D 21, 3305 (1980).
[21] S. Coleman, Phys. Rev. D 15, 2929 (1977).

[22] R. Basu, A. Guth, and A. Vilenkin, Phys. Rev. D 44, 340 (1991).

[23] J. Garriga, Phys. Rev. D 49, 6327 (1994).

[24] K. Lee and E. Weinberg, Phys. Rev. D 36, 1088 (1987).

[25] J. D. Brown and C. Teitelboim, Nucl. Phys. B279, 787 (1988).

[26] T. Banks, M. Dine, and L. Motl, J. High Energy Phys. 01, 031 (2000).

[27] A. Vilenkin, Phys. Rev. D 27, 2848 (1983).

[28] A. Linde, Phys. Lett. B 175, 395 (1986).

[29] V. Vanchurin, A. Vilenkin, and S. Winitzki, Phys. Rev. D 61, 083507 (2000).

[30] J. Garriga and A. Vilenkin, Phys. Rev. D 57, 2230 (1998).

[31] T. Banks, Phys. Rev. Lett. 52, 1461 (1984).

[32] G. W. Gibbons and S. W. Hawking, Phys. Rev. D 15, 2752 (1977).

[33] J. Garriga, Phys. Rev. D 49, 5497 (1994).

[34] J. Garriga and A. Vilenkin, Phys. Rev. D 44, 1007 (1991); 45, 3469 (1992).

[35] S. Giddings and A. Strominger, Nucl. Phys. B306, 890 (1988); K. Lee, Phys. Rev. Lett. 61, 263 (1988); L. F. Abbot and M. B. Wise, Nucl. Phys. B325, 681 (1989).

[36] R. Kallosh, A. Linde, D. Linde, and L. Susskind, Phys. Rev. D 52, 912 (1995).

[37] P. G. O. Freund and M. Rubin, Phys. Lett. 97B, 233 (1980); P. G. O. Freund, Nucl. Phys. B209, 146 (1982); N. Deruelle, J. Garriga, and E. Verdaguer, Phys. Rev. D 43, 1032 (1991).

[38] S. Weinberg, Dreams of a Final Theory (Pantheon, New York, 1992).

[39] J. Garriga, T. Tanaka, and A. Vilenkin, Phys. Rev. D 60, 023501 (1999).

[40] A. Vilenkin and S. Winitzki, Phys. Rev. D 55, 548 (1997).

[41] M. Tegmark and M. Rees, Astrophys. J. 499, 526 (1998).

[42] E. Witten, hep-ph/0002297.

[43] G. Dvali and G. Gabadadze, Phys. Rev. D 63, 065007 (2001).

[44] E. Guendelman and A. Kaganovich, Phys. Rev. D 60, 065004 (1999).

[45] G. Dvali and A. Vilenkin, hep-th/0102142. 